\title{
Alignment determination of the Hayabusa2 laser altimeter (LIDAR)
}

\author{
Hirotomo Noda ${ }^{1,21 *}$ (D), Hiroki Senshu², Koji Matsumoto ${ }^{1,21}$, Noriyuki Namiki ${ }^{3,21}$, Takahide Mizuno ${ }^{4,21}$, \\ Seiji Sugita ${ }^{2,5}$, Shinsuke Abe ${ }^{6}$, Hiroshi Araki ${ }^{3,21}$, Kazuyoshi Asari ${ }^{1}$, Yuichiro Cho ${ }^{5}$, Atsushi Fujiii, \\ Masahiko Hayakawa ${ }^{4}$, Arika Higuchi ${ }^{7}$, Naoyuki Hirata ${ }^{8}$, Naru Hirata ${ }^{9}$, Chikatoshi Honda ${ }^{9}$, Rie Honda ${ }^{10}$, \\ Yoshiaki Ishihara ${ }^{11}$, Shingo Kameda ${ }^{12}$, Shota Kikuchi ${ }^{4}$, Toru Kouyama ${ }^{13}$, Moe Matsuoka ${ }^{4}$, Yuya Mimasu4, \\ Tomokatsu Morota ${ }^{5}$, Satoru Nakazawa ${ }^{4}$, Kazunori Ogawa ${ }^{11}$, Naoko Ogawa ${ }^{4}$, Go Ono ${ }^{19}$, Shoko Oshigami , \\ Takanao Saiki ${ }^{4}$, Naoya Sakatani ${ }^{12}$, Sho Sasaki ${ }^{14}$, Hirotaka Sawada ${ }^{4}$, Makoto Shizugami ${ }^{3}$, Hidehiko Suzuki ${ }^{15}$, \\ Tadateru Takahashi ${ }^{4}$, Yuto Takei ${ }^{4,19}$, Satoshi Tanaka ${ }^{4,21}$, Eri Tatsumi ${ }^{5,16}$, Fuyuto Terui ${ }^{4}$, Yuichi Tsuda ${ }^{4,21}$, \\ Seiitsu Tsuruta', Sei-ichiro Watanabe ${ }^{4,17}$, Manabu Yamada², Ryuhei Yamada9 ${ }^{9}$, Tomohiro Yamaguchi ${ }^{18}$, \\ Keiko Yamamoto ${ }^{3}$, Yasuhiro Yokota ${ }^{4}$, Fumi Yoshida ${ }^{7}$, Kent Yoshikawa ${ }^{19}$, Makoto Yoshikawa ${ }^{4,21}$ \\ and Kazuo Yoshioka ${ }^{20}$
}

\begin{abstract}
In this study, we determined the alignment of the laser altimeter aboard Hayabusa2 with respect to the spacecraft using in-flight data. Since the laser altimeter data were used to estimate the trajectory of the Hayabusa2 spacecraft, the pointing direction of the altimeter needed to be accurately determined. The boresight direction of the receiving telescope was estimated by comparing elevations of the laser altimeter data and camera images, and was confirmed by identifying prominent terrains of other datasets. The estimated boresight direction obtained by the laser link experiment in the winter of 2015, during the Earth's gravity assist operation period, differed from the direction estimated in this study, which fell on another part of the candidate direction; this was not selected in a previous study. Assuming that the uncertainty of alignment determination of the laser altimeter boresight was 4.6 pixels in the camera image, the trajectory error of the spacecraft in the cross- and/or along-track directions was determined to be $0.4,2.1$, or $8.6 \mathrm{~m}$ for altitudes of 1, 5 , or $20 \mathrm{~km}$, respectively.
\end{abstract}

Keywords: Hayabusa2, LIDAR, Laser altimeter, Alignment determination, Field of view

\section{Introduction}

Precise determination of the alignment of a laser altimeter with respect to spacecraft is indispensable for the accurate estimation of the footprint positions on the surface of a celestial body for lunar and planetary missions. For example, in the SELENE (Kaguya) lunar exploration program, which had spanned from 2007 to 2009,

\footnotetext{
*Correspondence: hirotomo.noda@nao.ac.jp

${ }^{1}$ National Astronomical Observatory of Japan, Oshu, Iwate 023-0861, Japan

Full list of author information is available at the end of the article
}

spacecraft orbits were determined using two-way range and range-rate measurement of radio waves from the Earth, four-way Doppler measurement with a relay satellite, and the Very-Long-Baseline Interferometry (VLBI) tracking with multiple ground VLBI stations (Goossens et al. 2011). Assuming that these estimated orbits are true, the surface topography of the Moon was calculated as the vector summation of two vectors from the ground to the spacecraft and from the spacecraft to the surface footprint of the altimeter with the aid of spacecraft attitude (e.g., Araki et al. 2009; Noda et al. 2008). In this analysis, it is assumed that the position of the body 
is accurately described with the ephemeris and that the accuracy of orbit determination was better than that of the celestial body size.

The Hayabusa2, an asteroid sample-return spacecraft, was launched on December 3, 2014 and arrived at the target asteroid 162173 Ryugu on June 27, 2018. In the case of Hayabusa2, however, the initial trajectory determined from range and range-rate measurements by radio-tracking from the Usuda Deep Space Center on Earth of the Japan Aerospace Exploration Agency (JAXA) may contain an error in the order of hundreds of meters. This is because scientific observations are normally carried out when the spacecraft is not visible from the Earth. This makes radio-tracking data unavailable, because the communication high-gain antennas are fixed on the $+Z$ plane of the spacecraft, while the scientific instruments are mounted on the opposite side on the $-Z$ plane. Simultaneous observations were conducted in limited cases. Therefore, two methods for trajectory estimation were used. In the first method, the spacecraft trajectory is estimated as a by-product when a shape model is created with camera image data. These image data must be well scaled to the shape model, such that the spacecraft position at the time of imaging is also determined. The second is to adjust the spacecraft positions such that topographic features contained in tracks of the laser altimeter (called Light Detection and Ranging (LIDAR) for the Hayabusa2 mission) fit to the global shape model that exists at the time of estimation (Matsumoto et al. 2020). The latter is useful for longer-period orbit determination, because typically the cadence of image acquisition is relatively low due to the data downlink budget. In this method, the accuracy of trajectory is dependent on the accuracy of the alignment determination of the altimeter with respect to the spacecraft.

The laser link experiment conducted in the winter of 2015 , in which the laser pulses from the satellite laser ranging stations on Earth (Mount Stromlo Observatory, Australia and Koganei station of the National Institute of Information and Communications Technology (NICT), Japan) were detected by the LIDAR, aimed to determine the boresight direction of the receiving telescope of the LIDAR (Noda et al. 2017). In this experiment, the spacecraft attitude was changed in steps of $1 \mathrm{mrad}$ for the spiral search, while laser pulses were sent from the ground-based laser station toward the spacecraft, with the LIDAR waiting for these pulses. During the two periods, the LIDAR successfully detected laser pulses, and the data enabled the estimation of the boresight direction with a $1 \mathrm{mrad}$ uncertainty. This level of uncertainty is not negligible, because it amounts to an uncertainty of $20 \mathrm{~m}$ in the footprint position on the asteroid surface when the spacecraft is $20 \mathrm{~km}$ away from the asteroid (referred to as the "home position" of the spacecraft) and the diameter of the asteroid is $1 \mathrm{~km}$. This number may be immediately converted to the spacecraft position uncertainty. The uncertainty is smaller than that of the range and range-rate trajectory determination. However, when the footprints of other instruments are determined by applying this LIDAR-determined trajectory, these footprint positions are also impacted by the same error magnitude. This study attempts to constrain LIDAR alignment by using distinctive surface features of the asteroid taken by the altimeter and an imaging camera. A detailed description of the precise orbit determination by LIDAR data is found in Matsumoto et al. (2020). This study focuses on the alignment determination of LIDAR.

\section{Method}

We used time-series topographic data of LIDAR (Mizuno et al. 2017) and images taken by the Optical Navigation Camera-Telescopic (ONC-T) (e.g., Suzuki et al. 2018). A global shape model constructed from ONC images is also utilized to simulate elevation maps of the region within the ONC-T field of view. First, we estimated the LIDAR boresight direction by comparing the LIDAR topography with the simulated elevation maps. Then, the boresight was validated with images obtained at lower altitude. The details of this method are described below.

\section{LIDAR}

The LIDAR ranges the distance between the surface and the spacecraft by measuring the time of laser light emission and reception. For the LIDAR on Hayabusa2, the transmitter field of view is $2.4 \mathrm{mrad}$ and the field of view of the long-range receiving telescope is $1.5 \mathrm{mrad}$. It is unusual for the transmitter to have a wider beam divergence than the receiver for spacecraft laser altimeters. The original design value of the beam divergence was smaller than 1.5 mrad. However, during the development of the instrument, the beam profile was divided into two by multi-mode oscillation. Thus, the shape of the laser beam pattern and the position of the highest beam intensity differs from shot to shot. After confirming that the instrument can be used to measure the distance to the surface within the required range up to $25 \mathrm{~km}$, there was no further effort to improve the divergence angle of the transmitter to maintain the launch schedule (Mizuno et al. 2017). Therefore, in Hayabusa2 LIDAR, any part of the topographic features in the field of view of the longrange receiving telescope returns a range if the return level exceeds the detector threshold level. However, we do not have a specific value describing which part of the field of view was effective to provide a range value for two key reasons: (i) the waveform of the return pulse was an integration of the beam profile and topography acquired 
with an electric integrator circuit, and (ii) the beam pattern differed from shot to shot and the position of the highest intensity may be stochastically distributed.

We converted the time-series range data of LIDAR into topography expressed in an asteroid-fixed frame (the definition is described in Sugita et al. 2019). This was carried out after estimating the spacecraft trajectory using the LIDAR measurements. We adjusted spacecraft positions such that convex or concave topographic features contained in the LIDAR tracks were fit to the existing global shape model (Matsumoto et al. 2020). The correction term with respect to the given initial trajectory was expressed by a polynomial function of time, and we searched for the best correction that minimized the discrepancy using the Markov chain Monte Carlo (MCMC) method. Matsumoto et al. (2020) used the home position keeping (HPK) trajectory routinely provided by the Hayabusa 2 engineering team as the initial trajectory. However, for the current analysis of data from mid-altitude operation (see subsection Selected data and comparison method), we utilized another image-based trajectory also provided by the engineering team during this special science observation period. Although the image-based trajectory was better than the HPK trajectory and the same method as Matsumoto et al. (2020) was applied in this study, the resultant footprint position may be less accurate than that in Matsumoto et al. (2020). This is because the method relies on along-track topographic information, i.e., it is difficult to retain a sufficiently long unperturbed arc due to more frequent thrusting to maintain the spacecraft altitude between 5.1 and $5.2 \mathrm{~km}$.

\section{ONC-T}

The ONC-T is an imaging camera in the visible wavelength whose instantaneous field of view is $0.1074 \mathrm{mrad}$ and the number of pixels $1024 \times 1024$. Therefore, the total field of view is $0.11 \times 0.11 \mathrm{rad}$. Image viewing and area drawing were undertaken using a software for astronomy: SAO Image DS9. The coordinate definition of the images is explained in Fig. 1. The center of the upper-left pixels is $(X, Y)=(1,1)$, with the $+X$ axis on the right and $+Y$ axis downward. In the ONC-T image, prior to the end of 2018, the $+X$ direction was the same as the longitudinal direction of the asteroid, and $-Y$

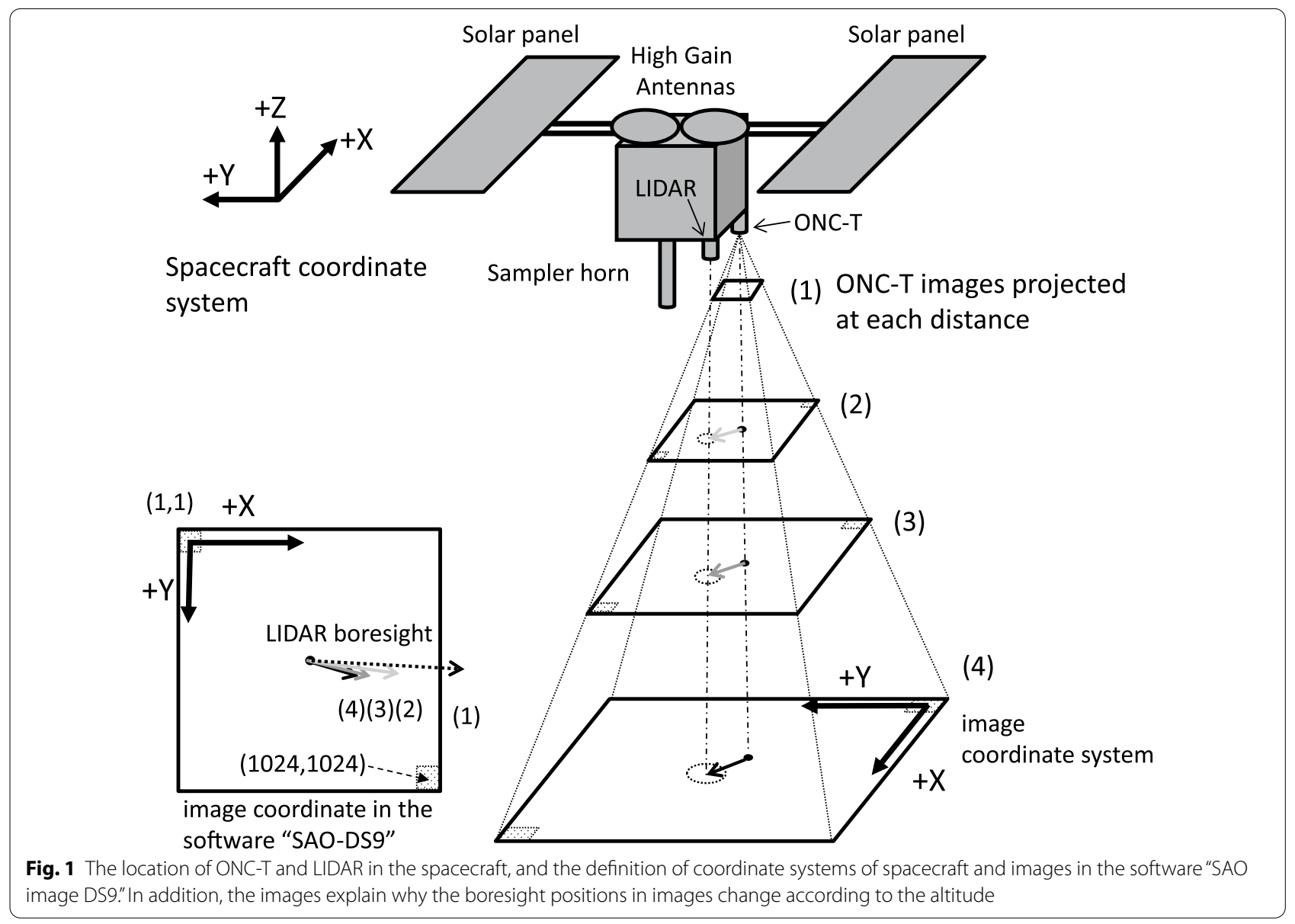


corresponded to a positive latitudinal direction. In 2019, the $-X$ direction was in the positive longitudinal direction and $+Y$ in the positive latitudinal direction, because of the spacecraft yaw-around operation at the end of 2018. This means that images must be flipped accordingly in the viewing software depending on when they have been acquired. If the images are flipped correctly, the surface terrain moves from left to right in the ONC-T images due to the asteroid rotation, and central longitude in the images decreases as time evolves.

The pixel coordinates of the boresight of LIDAR in images vary based on the altitude above the asteroid surface, as the locations of these two instruments differ by 1.360 and $0.235 \mathrm{~m}$ in the $X$ and $Y$ direction, respectively, in the spacecraft frame (i.e., there is a parallax effect shown in Fig. 1). We developed a simple simulation code to predict the location of the LIDAR footprint position in the ONC-T image with respect to the specified altitude. For example, based on the simulation, the pixel position in the $\mathrm{X}$ direction at a 1.6 or $5 \mathrm{~km}$ altitude differs by 8.5 or 2.2 pixels, respectively, compared to that at a $20 \mathrm{~km}$ altitude. As such, in the alignment determination trial, it is necessary to limit the time period of the data if the same value of the boresight position is used during descent.

\section{Shape model and simulated elevation map}

To identify the LIDAR boresight position in the ONC-T images, we compared LIDAR-observed topography with elevation information within the camera field of view. This is a map with elevation values corresponding to each of the ONC-T image pixels. The elevation was defined as the radial distance from the asteroid center to the surface point. Such an elevation map may be simulated by a ray tracing method when a shape model, spacecraft position and attitude at specific ONC-T observation epoch, and camera parameters are provided. We used the shape model SHAPE_SPC_3M_v20200323.bds where three million facets were reconstructed from multiple ONC images. This is an updated version of the model presented by Watanabe et al. (2019). We used the spacecraft position and attitude data obtained through the shape modeling process as the adjusted camera position and direction. This was because this by-product information was estimated such that the images were best registered. The accuracy of image registration at an altitude of approximately $5 \mathrm{~km}$ was better than 1 pixel, corresponding to $0.5 \mathrm{~m}$ on the surface.

\section{Selected data and comparison method}

As the transmitted beam intensity of LIDAR differs from shot to shot, we attempted to identify the location in the field of view of the long-range receiving telescope. This statistically provides a range using a large amount of data taken at an approximately constant altitude. Then we confirmed the expected boresight using a small amount of data taken at a lower altitude. Finally, we converted the boresight in the ONC-T image to the spacecraft coordinate system.

Since June 27, 2018, when the spacecraft had commenced proximity observation of the asteroid, several lower-altitude observations were carried out to prepare for surface material sampling. First, we estimated LIDAR boresight direction with the data of the midaltitude observation conducted on August 1, 2018, when spacecraft altitude was maintained at $5.1-5.2 \mathrm{~km}$ for one rotation of the asteroid, observing nadir direction without the scanning spacecraft attitude. The 147 ONC-T images were obtained between $14 \mathrm{~h} 10 \mathrm{~m}$ $45 \mathrm{~s}$ and $22 \mathrm{~h} 25 \mathrm{~m} 01 \mathrm{~s}$, while the LIDAR observed the range with a repetition rate of $1 \mathrm{~Hz}$. As the field of view size of the LIDAR is 14 pixels of the ONC-T, the elevation within the search area of $50 \times 50$ pixels near the image center of each simulated elevation map was compared with the LIDAR-observed elevation to determine whether these two values match within the given tolerance. We then obtained a frequency distribution map to identify how many of the 147 simulated elevation maps matched with the LIDAR-observed elevation for each pixel. We set a 2-m tolerance for elevation comparison between simulated elevation images and LIDAR-observed topography, because the mean value of the spacecraft trajectory residual when the LIDAR topography was successfully fitted to a shape model was approximately $2 \mathrm{~m}$ (Matsumoto et al. 2020).

We then validated this value with data at loweraltitude operation. For this purpose, we used the time period when characteristic convex terrains were obtained during the descent operation for gravity estimation in $22 \mathrm{~h}$ on August 6, 2018. The altitude we focused on was approximately $1.5 \mathrm{~km}$. At this altitude, the diameter of the LIDAR laser footprint was approximately $2.3 \mathrm{~m}$, while the average area of one surface of the global shape model with 3 million polygons was $\sim 1 \mathrm{~m}^{2}$; the spatial resolution of the shape model was not sufficient. To address this, we created a local digital elevation model (DEM) and established similar topographic maps to compare the LIDAR topography. However, the local DEM did not successfully reproduce the edge of the boulders as the surrounding topography had become smoother than the LIDAR topography. This means that the LIDAR topography could not be reproduced even by using the local DEM. As such, we tested whether the previously determined pixel position was correct using only the ONC-T image data. 
We also analyzed the data when LIDAR irradiated a Target Marker for a 2-s duration at $01 \mathrm{~h} 59 \mathrm{~m} 51.4 \mathrm{~s}$ and $01 \mathrm{~h} 59 \mathrm{~m} 52.4 \mathrm{~s}$ at an altitude of $75 \mathrm{~m}$ on June 13, 2019, in Appendix.

\section{Results}

Statistical analysis of simulated elevation maps

Figure 2 illustrates an example of a simulated elevation map from the shape model (Fig. 2a, c) and an ONC-T image taken at $14 \mathrm{~h} 10 \mathrm{~m} 45 \mathrm{~s}$ on August 1, 2018 (Fig. 2b, d). The red boxes in Fig. 2a, b correspond to the
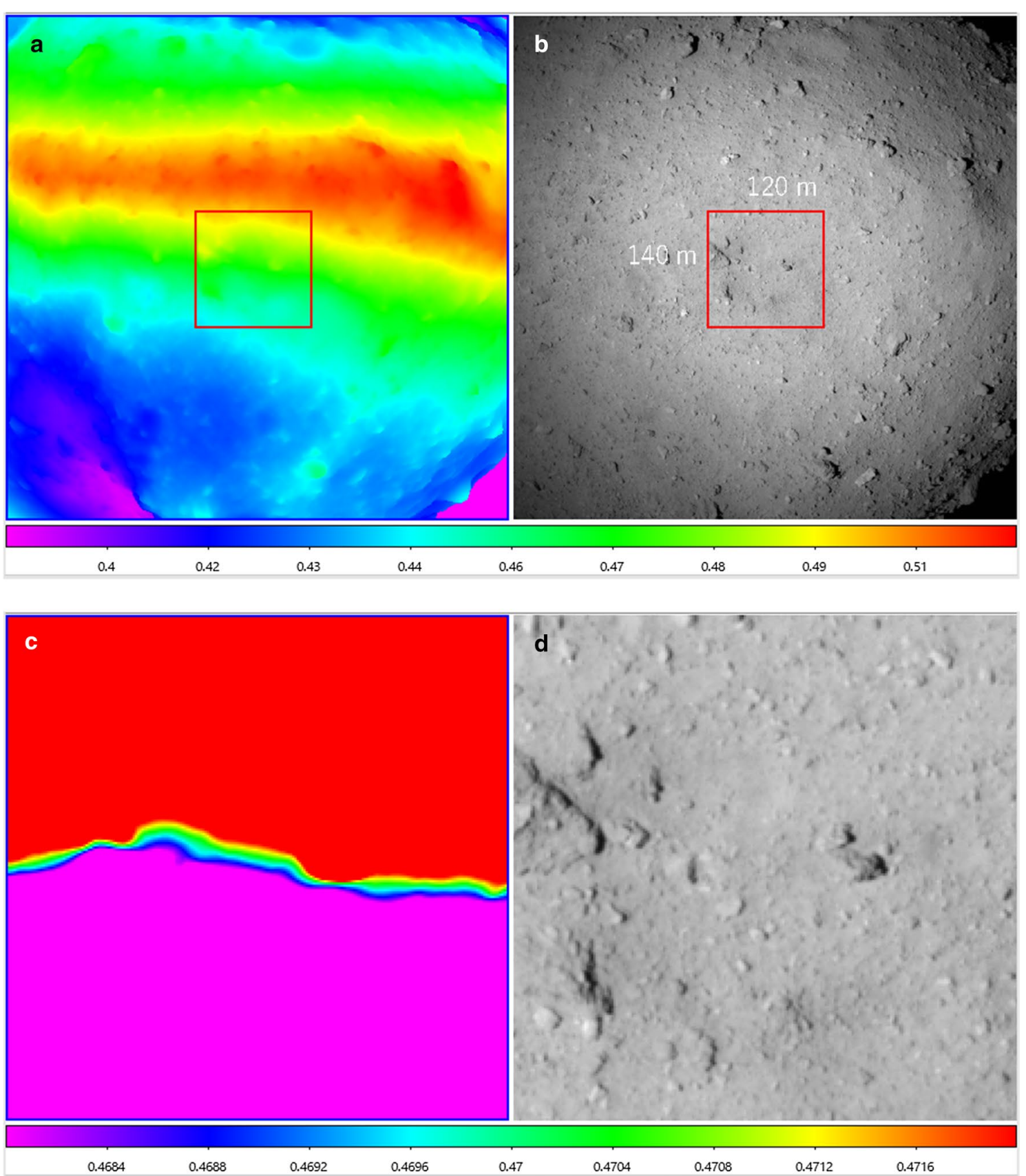

Fig. 2 An example of a simulated elevation map made from the shape model $(\mathbf{a}, \mathbf{c})$ and the corresponding ONC-T image (b, d). The color bars in the bottom of $(\mathbf{a}, \mathbf{c})$ show the radial distance from the center for $(\mathbf{a}, \mathbf{c})$, respectively. The unit is $\mathrm{km}$. In $(\mathbf{b})$, the latitude range of $30 \mathrm{~N}$ and $50 \mathrm{~S}$ and longitude of $150 \mathrm{E}$ to $250 \mathrm{E}$ are covered, and the asteroid limb is seen in the bottom right. The red boxes in $(\mathbf{a}, \mathbf{b})$ correspond to the area shown in (c, d). (image list: hyb2_onc_20180801_141045_tvf_I2a.fit) 
area shown in Fig. 2c, d. In Fig. 2c, the color scale was adjusted such that the LIDAR topography $(0.4699 \mathrm{~km})$ was included in the area between purple and yellow, highlighting a 2-m tolerance range. Figure 2c shows that a single elevation map has many pixel positions where the elevation value is within the LIDAR-observed elevation with a given tolerance. Pixels within the search area of $50 \times 50$ pixels were selected as candidate locations where the LIDAR measured the range. When we use many elevation maps, the candidate pixel position of the LIDAR field of view may be narrowed as the topography differs by map. Figure 3 presents the results of the frequency distribution of elevation matching. The horizontal and vertical axes correspond to $X$ and $Y$, respectively, of the ONC-T images where the unit is pixels. Note that in the vertical axis, downward is the positive direction; this is the same manner of expression as the SAO image DS9. We obtained the $(X, Y)=(487,498)$ pixel position as the most probable value for this altitude, in which the pixel simulated elevation matches the LIDAR elevation within a given tolerance for 81 of 147 images. Neighboring pixels also show the same frequency level, although the contours show that the match cases decrease with increasing distance from the peak. Based on the premise that the field of view diameter is 14 pixels, if the topography is sufficiently random, a circular area with a 14-pixel diameter will appear. In this case, however, as shown in Fig. 2, the topography with the same contour level is horizontally elongated as it is located in the equatorial bulge;

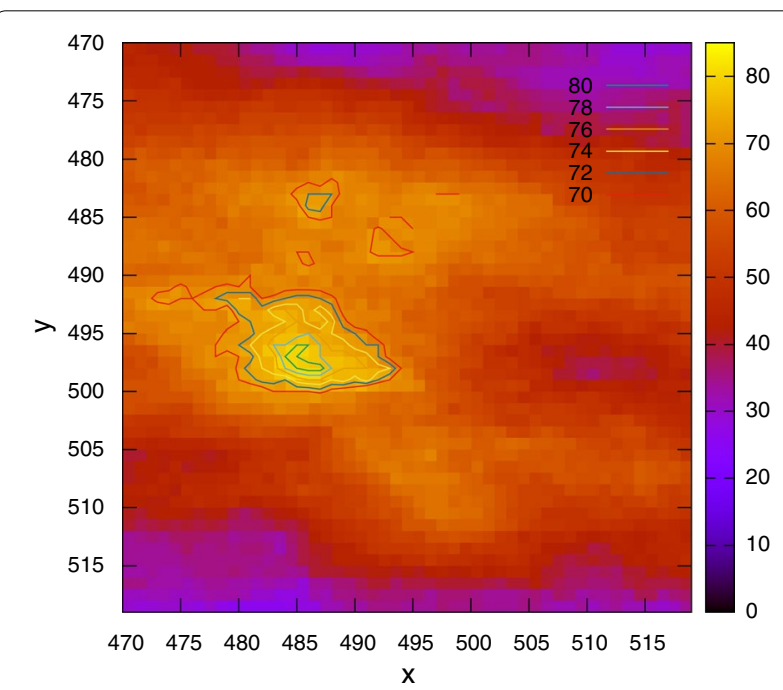

Fig. 3 Graph showing the frequency distribution with regard to the pixels in how many images the topographic data (elevation) from the shape model match the LIDAR-observed elevation. Horizontal and vertical axes correspond to $X$ and $Y$ direction of ONC-T images. The color bar shows the occurrence frequency. The unit is the number of images this result is also impacted by such terrain features. However, as the contour level " 70 " is almost the same size as the field of view size in the horizontal direction, the peak location selected was located near the center. The estimation uncertainty of the center may be a few pixels, almost equivalent to $1 \mathrm{~m}$.

\section{Validation with data at lower altitude}

We attempted to validate the value selected above using the other dataset. Figure 4 presents the time-series LIDAR topography obtained for $40 \mathrm{~min}$ from $21 \mathrm{~h} 50 \mathrm{~m}$ to $22 \mathrm{~h} 30 \mathrm{~m}$ on August 6, 2018, together with two ONC-T images (taken at $21 \mathrm{~h} 58 \mathrm{~m} 49 \mathrm{~s}$ and $22 \mathrm{~h} 21 \mathrm{~m} 48 \mathrm{~s}$ ). This was aligned to a shape model and the LIDAR track using a mapping tool: the Small Body Mapping Tool (Ernst et al. 2018). The grid size was $2^{\circ}$ in both the longitudinal and latitudinal directions. We selected this period as two distinctive convex topographic profiles were prominent, such as the short passage of a boulder and two consecutive boulders denoted as thick black bars in Fig. 4. The LIDAR range changed by $415 \mathrm{~m}$ (from 1740 to $1325 \mathrm{~m}$ ) during this period, and the LIDAR footprints move in the longitudinal direction due to asteroid rotation. In Fig. 4, time evolves from right to left such that the longitude direction of the images and time-series topographic data match. In the upper panel, the filled green circles in the LIDAR data indicate times when the ONC-T image data were taken. The vertical axis represents the radial distance from the asteroid center. Eleven prominent convex topographic features exist in the LIDAR topography and may be associated with the boulders in the images. Subsequently, we used two time periods denoted by the thick black bars in the topographic profile.

We first focused on "time period 9" which has a $14 \mathrm{~s}$ duration between $22 \mathrm{~h} 22 \mathrm{~m} 10.9 \mathrm{~s}$ and $22 \mathrm{~h} 22 \mathrm{~m} 23.9 \mathrm{~s}$ in Fig. 4, when the LIDAR topographic data showed a sudden and short-period increase with $8 \mathrm{~m}$. This enhancement reflects the passing of the boulder 9. We created a local DEM to demonstrate whether the LIDAR topography may be reproduced with this DEM. Although the center position was scanned by $50 \times 50$ pixels, the LIDAR terrain could not be reproduced by the local DEM. Therefore, only the image data were used to confirm the estimation. Figure 5 shows the collection of images and LIDAR topography when ONC-T captured the boulder 9 image at $22 \mathrm{~h} 22 \mathrm{~m} 21.9 \mathrm{~s}$ before and after. The format of the time-series topography is the same as in Fig. 4. The green circles have a 14-pixel diameter, expressing the field of view of the receiving telescope of LIDAR which is $1.5 \mathrm{mrad}$. All circles in these images have the same size and location. At $22 \mathrm{~h} 22 \mathrm{~m} 21 \mathrm{~s}$, the field of view was on the horn-like tip of the boulder, while the two images before and after barely touched the edge of the boulder. 

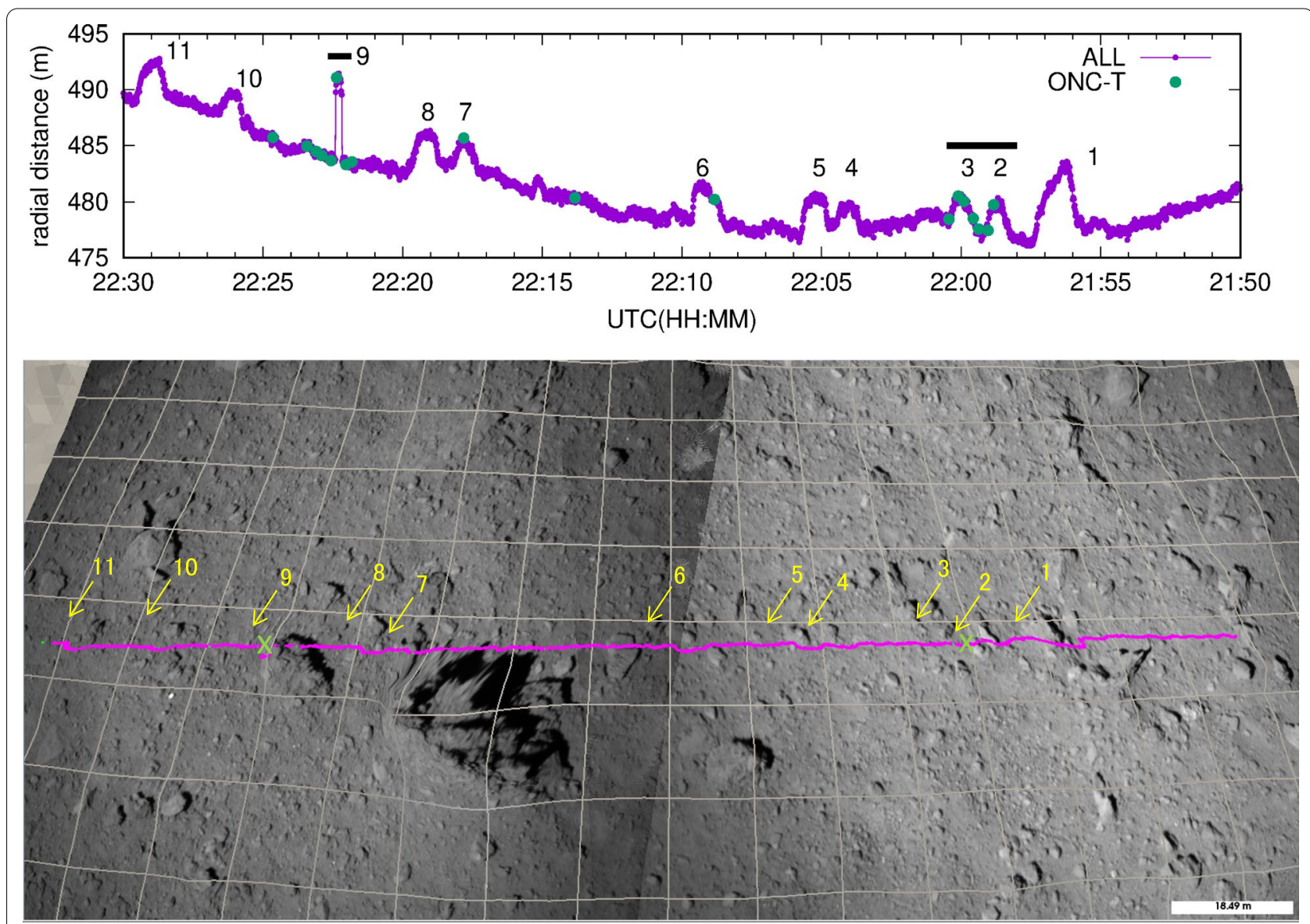

Fig. 4 Selected time-series LIDAR topography and image data on August 6, 2018 for alignment determination. Black thick bars in the top panel denote the time periods we focused on. Images are mapped onto the shape model with a mapping tool with $2^{\circ}$ longitudinal and latitudinal grid. Approximate LIDAR footprints are plotted at the bottom figure, but part of them appear to be offset downward due to convex terrain. Approximate two LIDAR footprint positions when these images were taken are marked by crosses. (Image list: hyb2_onc_20180806_215849_tvf_I2a.fit, hyb2_ onc_20180806_222148_tvf_I2a.fit)

The same procedure was used for the areas of boulders 2 and 3; the results are shown in Fig. 6. The enlarged time-series graph of the topographic profile was placed in the center surrounded by ONC-T images taken at each time, describing how surface topography moves against the fixed green circle (estimated LIDAR field of view) in each image. The circles were drawn at $(X, Y)=(492,499)$ for this altitude. The format of the time-series topography in the center was the same as in Fig. 4. The topographic profile was retrieved by the area covered with green circles; if boulders are located in the circle, the topography returns a higher value, while the reverse is also true. Using this process, we confirmed that the boresight direction determined for an altitude of $5.1 \mathrm{~km}$ on August 1, 2018, also performed well for images in Fig. 6. This was based on the fact that a small shift in the $X$ direction (horizontal) could easily change topography within the green circle in this dense boulder constellation.

Then, we converted the boresight position in the image to the spacecraft coordinate system using information on the ONC-T fitting angle in the spacecraft. The LIDAR boresight vector in the spacecraft-fixed coordinate system was $(0.003976123210772,0.000867844437128$, 0.999991718610832). Figure 7 presents this value and another by Matsumoto et al. (2020), together with one obtained by a laser link experiment (Noda et al. 2017). Since the boresight direction was almost aligned in the $-Z$ direction, only the $X$ - and $Y$-values were plotted in mrad unit in Fig. 7. In this figure, the black solid or dotted circles are the field of view areas and their centers represent the estimated boresight. The points indicate the direction where laser pulses from the ground-based 


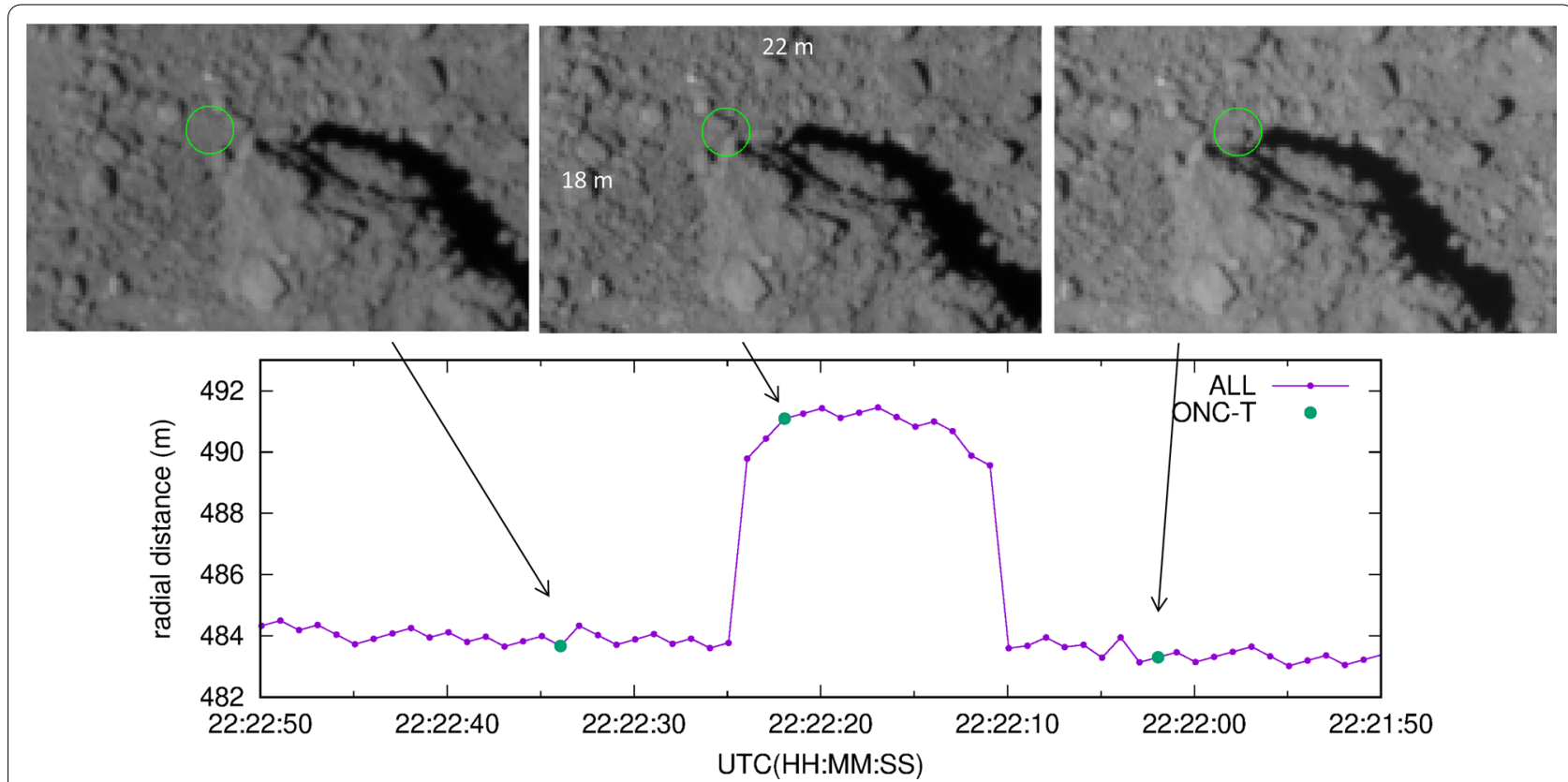

Fig. 5 ONC-T images when the LIDAR field of view passed boulder 9. The open green circles in images have 14-pixel diameter which corresponds to the same size as the field of view size of the long-range receiving telescope of LIDAR (1.5 mrad). The centers of the circle are drawn at (492, 499) in each image. (Image list: hyb2_onc_20180806_222201_twf_I2a.fit, hyb2_onc_20180806_222221_txf_I2a.fit, hyb2_onc_20180806_222233_tnf_ 12a.fit)

laser station were detected during the spacecraft attitude scan in the laser link experiment.

\section{Discussion}

\section{Boresight direction}

The boresight direction determined from the laser link experiment differed by $1.05 \mathrm{mrad}$ from the results of this study. This value was the same as the scan step size of the spacecraft attitude change in the laser link experiment (Fig. 7). As described by Noda et al. (2017), this area was selected as the circle was able to cover the area where all laser pulses from the Earth were detected (\#13 and \#30 on December 15 and \#31 and \#32 on December 11, 2015). However, the area where pulses were detected exceeds the nominal field of view of $1.5 \mathrm{mrad}$ of the receiving telescope. There are no good reasons for this; however, the bright rings outside the Airy disk may have been detected due to the effect of diffraction because of the strong laser pulses. We can conclude that the estimated direction used by Noda et al. (2017) could not retrieve the topographic profiles taken at $22 \mathrm{~h} 22 \mathrm{~m}$ on August 6, 2018 . This was because the field of view for this was not located on the horn-like shape topography shown as a white circle in Fig. 8, and thus, the passing boulder 9 could not be realized. Therefore, the value in this study was considered to outperform that of Noda et al. (2017).
Figure 9 provides an example of the alignment difference between this study and another by Matsumoto et al. (2020) in the image data taken at $07 \mathrm{~h} 52 \mathrm{~m} 08 \mathrm{~s}$ on July 20, 2018. The time-series topography in purple in Fig. 9a shows that the LIDAR measured a boulder with a radial distance of approximately $468 \mathrm{~m}$. Figure $9 \mathrm{~b}$, c represents the ONC-T image, and Fig. 9d shows the simulated elevation map with the same angle of view as Fig. 9c. The black and green circles in Figs. 9c, d indicate the estimated LIDAR field of view obtained by Matsumoto et al. (2020) and the one determined in this study, respectively. The green circle in Fig. 9d is located on a boulder colored red where the radial distance value was the same as that shown in Fig. 9a, while the black circle had a smaller radial distance. The maximum, average, and minimum values of the radial distance in the green circle of Fig. 9d were also plotted as dots in Fig. 9a, showing that the maximum value among the field of view matches the LIDAR-observed radial distance.

\section{Possible detection of diffracted light}

The size of the field of view of the receiving telescope was determined by a pinhole installed on the focal plane. The value of $1.5 \mathrm{mrad}$ was not a measured in the calibration test, rather, it was calculated based on the design. The error was approximately $0.1 \mathrm{mrad}$ despite considering the mounting tolerance. In the laser link experiment, the 


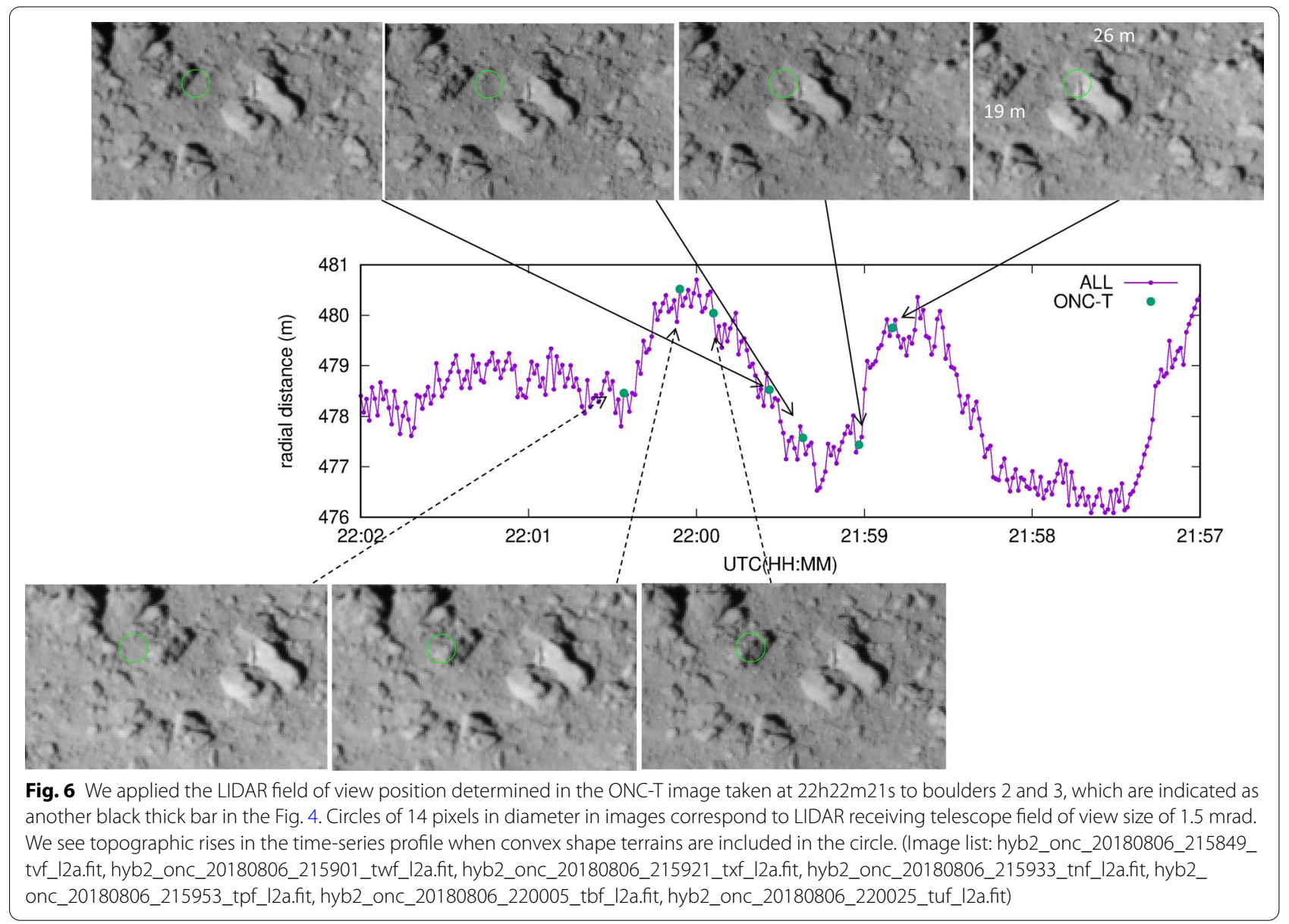

laser intensities received by the LIDAR were not measured when the spacecraft attitude scan was conducted; however, they were expected to be very high. The distances to the spacecraft at that time were approximately 3.3 Mkm (December 11, 2015) and 5.0 Mkm (December 15,2015$)$. When the distance to the spacecraft was 6.6 Mkm (December 19, 2015), the received light intensity was measured by setting the LIDAR in the range mode, whereby the received laser intensity was saturated or nearly saturated in some periods. Since the received light intensity was inversely proportional to the square of the distance in the one-way link, the received light intensity may have been 4 or 1.7 times the saturation level (1515.4 mV) on December 11 and December 15, 2015. The return light might form diffraction pattern (Airy pattern) outside the main beam known as the Airy disk. The intensity of the first bright ring of the Airy pattern is 1.7\% of that of the Airy disk, and the light-receiving threshold of LIDAR was set to $27 \mathrm{mV}$. For strong light, the first bright ring would be detected even if the disk was out of the field of view. On the contrary, if the received light intensity was not saturated, there was low potential for detecting diffracted light, making it unlikely that the field of view appeared wider than the nominal value at the home position and mid-altitude operations.

\section{Thermal effect}

It is conceivable that the alignment of optical instruments changes as the spacecraft structure expands or contracts due to variations in the thermal environment. Thus far, there has been no information that the field of view of the onboard optics has shifted due to thermal expansion or contraction of the spacecraft structure. This information is summarized below:

\section{a. Expected thermal expansion of spacecraft}

The spacecraft attitude was controlled such that the $+Z$ plane nearly faced the Sun, and the $+X$ plane was also slightly illuminated by the Sun besides the $+Z$ plane. According to the temperature history of some instruments inside the $+X$ plane, the temperature was within the thermostat control range 


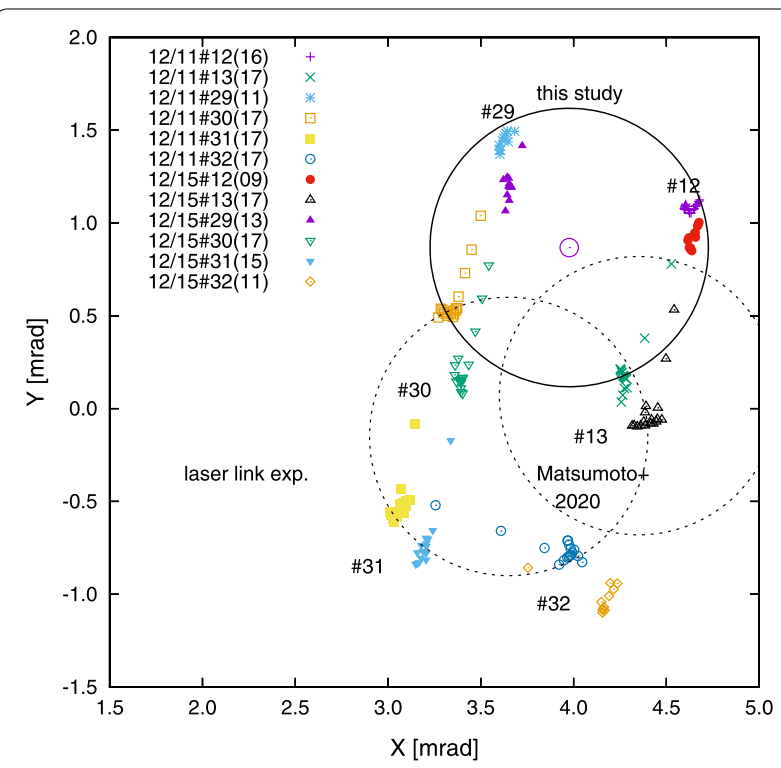

Fig. 7 A comparison of estimated LIDAR field of view areas in this study, the laser link experiment, and Matsumoto et al. (2020). A solid circle representing the result of this study is overlaid on the result of laser link experiment and Matsumoto et al (2020). The points indicate the direction where laser pulses from the ground-based laser station were detected in the laser link experiment. As a result, the field of view determined in this study lied on the other side of the results in the laser link experiment

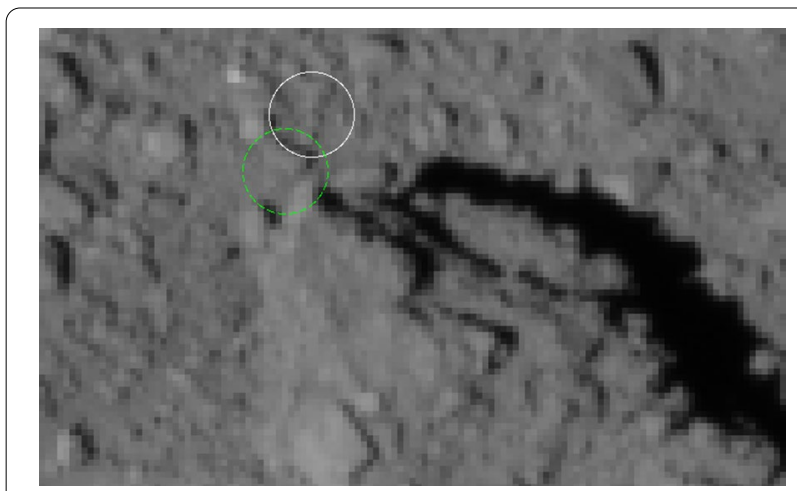

Fig. 8 White and green dashed circles are estimated LIDAR receiving telescope field of view from Noda et al (2017) and this study, respectively. The white circle is located out of the boulder, and the LIDAR observed topography on the passage of boulder 9 will not be retrieved. (Image list: hyb2_onc_20180806_222221_txf_I2a.fit)

with the exception of approximately three months near the perihelion (June 2018 and September 2019). The temperature deviation from the control range was approximately $10{ }^{\circ} \mathrm{C}$ or less. Assuming the temporal change of the $-X$ plane as $10^{\circ} \mathrm{C}$ and the coefficient of thermal expansion of aluminum as $2.3 \mathrm{E}-5$ $(/ \mathrm{K})$, the expected tilt angle of the $-Z$ plane (where LIDAR and ONC-T are mounted), caused by the temperature difference in $+X$ and $-X$ planes, was approximately $0.23 \mathrm{mrad}$. This value is sufficiently small for the LIDAR receiving telescope's field of view of $1.5 \mathrm{mrad}$. Most of the other time, including during the laser link experiment after the Earth gravity assist in 2015, the temperature was within the control range. As such, it is considered that the expansion or contraction of the structure from the normal state did not occur.

b. Temperature changes of the LIDAR

In a laser altimeter, the mutual alignment between the transmitting and receiving telescopes is important as return pulses travel outside of the field of view of the receiving telescope if these two telescopes are misaligned; this means that ranging is impossible. In the thermal vacuum test for the LIDAR prior to being mounted to the spacecraft, the mutual alignment measurement of the long-range receiving and transmitting telescope was carried out assuming the perihelion (hot) and aphelion (cold) cases. The difference in the mutual alignment between hot and cold cases was approximately $0.1 \mathrm{mrad}$ as the worst value. In orbit, the temperature was maintained within the control range of the cold case throughout the observation periods, and it was expected that the mutual alignment did not change significantly.

c. LIDAR boresight estimation near aphelion

For the confirmation described in (b), we verified the alignment near the aphelion. For example, at an altitude of $2.2 \mathrm{~km}$ during the descent operation at $21 \mathrm{~h} 12 \mathrm{~m} \mathrm{44}$ s on March 7, 2019, close to the aphelion, the alignment that had been estimated successfully identified a small boulder in the ONC-T image from a boulder-like topography measured with the LIDAR between $21 \mathrm{~h} 12 \mathrm{~m}$ and $21 \mathrm{~h} 13 \mathrm{~m}$ (Fig. 10). The boulder was located at a large impact crater at (longitude, latitude $)=\left(229.95^{\circ}, 3.24^{\circ}\right)$ with a $142-\mathrm{m}$ diameter; this was apparent as the downward slope in the time-series topography data. Therefore, it may be concluded that at least the same alignment information may also apply this time.

\section{Error evaluation}

The pixel reading error of images comprised a major part of the uncertainty of the boresight direction in this study. In reality, it is difficult to determine the actual amount of pixel reading error as the local DEM is unable to retrieve the topographic profile of LIDAR observations at lower altitudes. For example, in Fig. 5, we were unable to assume how the determined LIDAR field of view retrieved the observed 14 s convex topography for the horn-shaped terrain in the northern edge of the boulder. This was attributed to two key reasons: (i) we did not have a specific value 

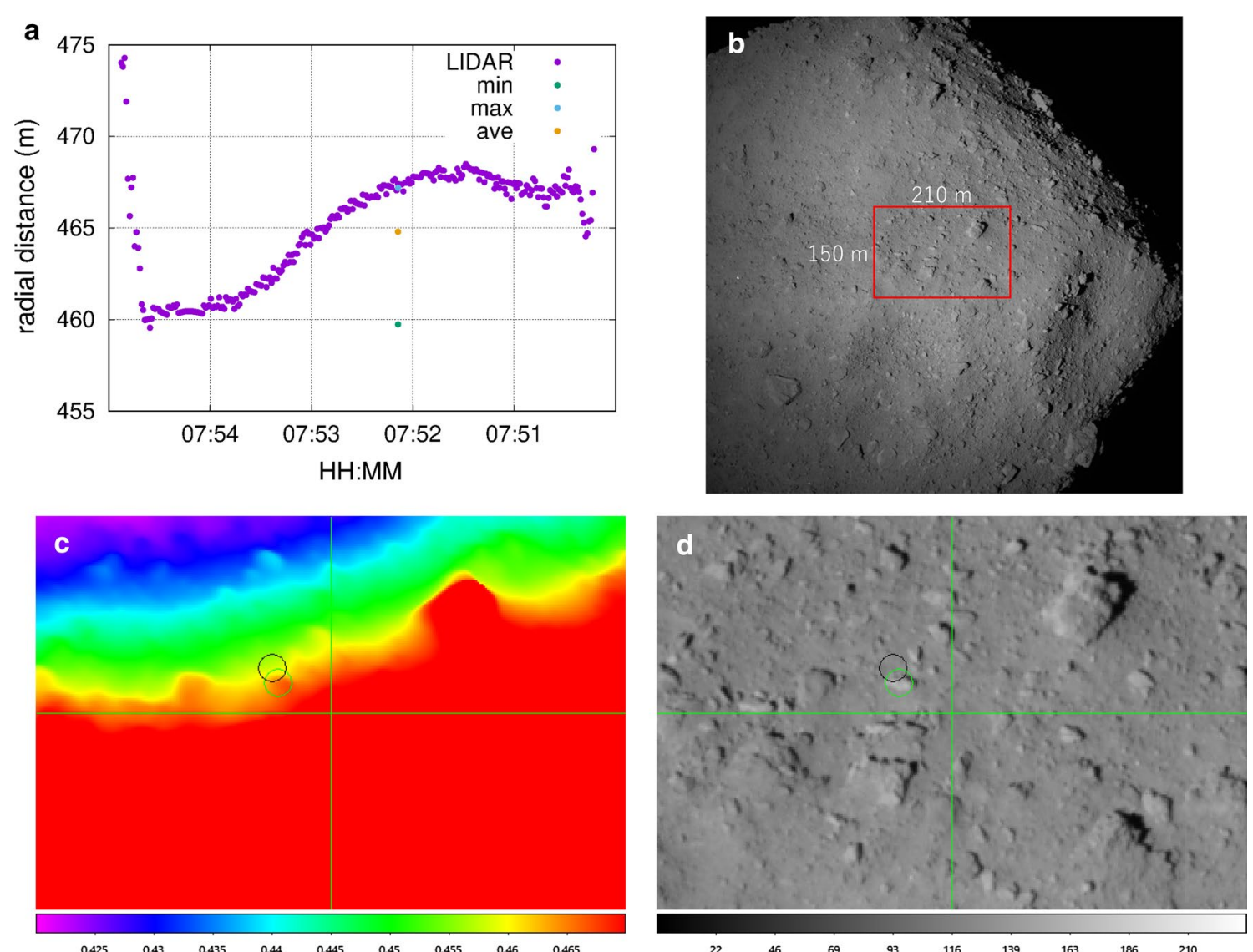

Fig. 9 a Top left. Comparison between LIDAR topography and maximum, minimum, and average topography in the simulated field of view of the LIDAR estimated from shape model in the green circle. btop right. Whole view of the ONC-T image taken 07h52m08s on July 20, 2018 at altitude of $6.4 \mathrm{~km}$ from the surface. The area in the red frame corresponds to the area shown in (c). c bottom right. Enlarged ONC-T image of (b). Black and green circle are estimated LIDAR receiving telescope field of view from Matsumoto et al (2020) and this study, respectively. $\mathbf{d}$ bottom left. Simulated topographic map from the shape model with the same viewing angle as (c). Meaning of the two circles are the same as (c). (Image list: hyb2_onc_20180720_075208_tvf_12a.fit)

describing how much part of the field of view was effective to return a range, and (ii) a DEM for this spatial scale was unavailable in this study. Therefore, we simply estimated the northern and southern limits of the possible location of the field of view based on two cases: (i) a 1 pixel-sized high topography moved for $14 \mathrm{~s}$ in the field of view, and (ii) horizontally distributed high topography was maintained at the edge of the field of view for $14 \mathrm{~s}$. As the horizontal speed of the field of view in this image is 0.719 pixels/s for this radial distance of the terrain $(484 \mathrm{~m})$ and LIDAR range $(1414 \mathrm{~m})$ with the asteroid rotation period $(7.63262 \mathrm{~h}), 10$ pixels of the higher terrain were allowed to be included in the field of view. These situations are summarized in Fig. 11. A yellow line that was 10 pixels in length touched the tip of the horn-like shaped terrain, inscribed by a yellow circle that corresponds to the northern limit case (i). The red line also contained 10 pixels, which is the northern (upward) boundary of the high terrain distributed horizontally. A red circle circumscribes the red line, showing that this is the southern (downward) limit of the possible field of view for case (ii). As a result, the possible $Y$ coordinate for the center of the field of view was located between the yellow and red circles, and ranged between 495 and 500, assuming that any part of the horn-like shape provided the observed high topography. Then, the uncertainty for this direction was set as 5 pixels, or \pm 2.5 pixels. The $X$ coordinate was not determined from this information. For the $X$ direction (right positive), based on the continuous images in Fig. 6, a 3-pixel shift in the $X$ direction would change the topographic profile significantly. For example, in the second image, the circle circumscribes a boulder on the right, and when the circle was shifted to the right, the resultant LIDAR topography must be different. In the third image, a 4-pixel shift to the left forces the boulder on the left to be within the circle. In the seventh image, it was expected that the shift to both right and left 

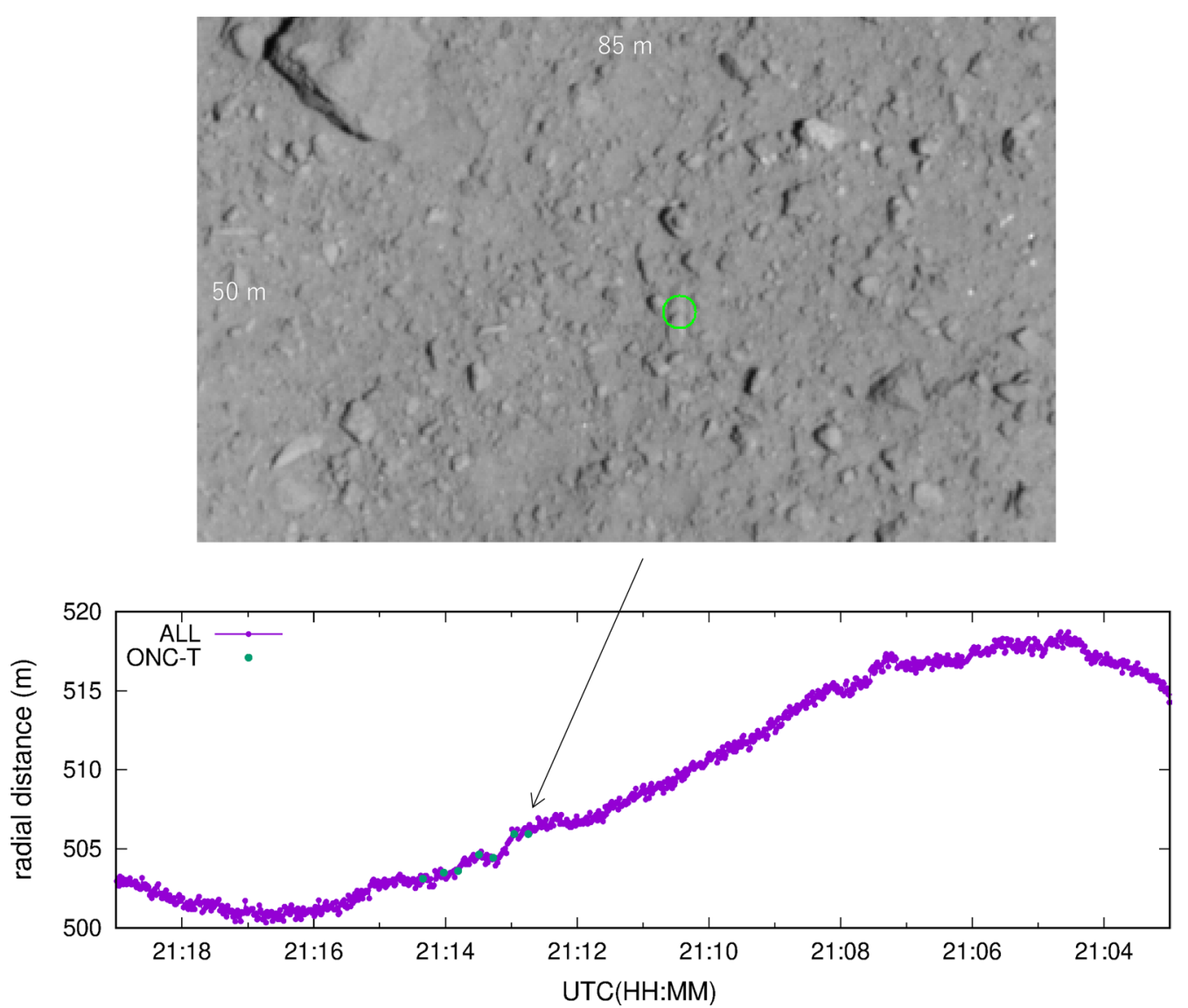

Fig. 10 A small boulder was identified in the image taken at 21h12m44s on Mar 7, 2019, close to the aphelion. The format of the photo (top) and the topography (bottom) is the same as in Fig. 9. (Image list: hyb2_onc_20190307_211244_tvf_I2a.fit)

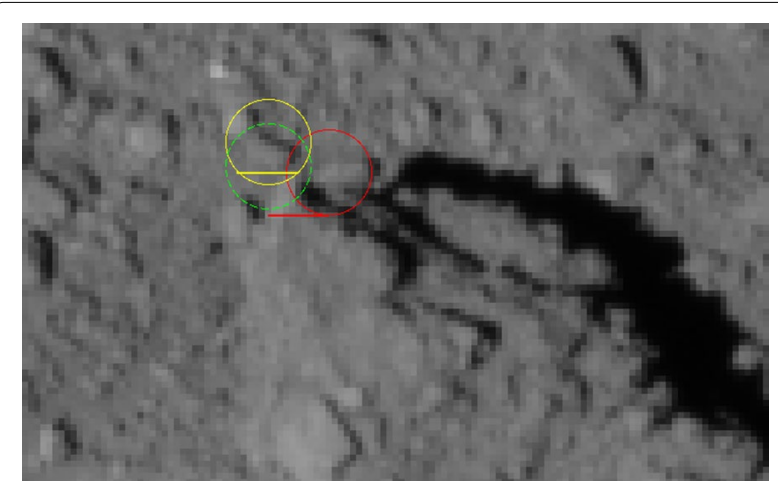

Fig. 11 A green dashed circle is the estimated FOV location for this altitude. A yellow line which touches the tip of the horn-like shaped terrain has 10 pixels, inscribed by a yellow circle centered at $(X, Y)=(492,495)$ with 7-pixel radius. A red line also contains 10 pixels, which is the northern (upward) boundary of the high terrain. A red circle circumscribes the red line, showing that this is the southern (downward) limit of the possible field of view. The $Y$ coordinate for the center of the yellow and white circles are 495 and 500 , respectively. 10-pixel lines correspond to the passing time of the boulder 9 for 14 s. (Image list: hyb2_onc_20180806_222221_txf_I2a. fit) may alter the LIDAR topography as the circle was located between boulders. If we take the root sum squared value of 4 pixels (square root of $2.5^{2}+3^{2}$ ) as the total pixel reading uncertainty, the uncertainty in the spacecraft trajectory, originating from the alignment estimation error at altitudes of 1,5 , or $20 \mathrm{~km}$, is $0.4,2.1$, or $8.6 \mathrm{~m}$ in the crossand/or along-track directions, respectively. Additionally, there is also uncertainty of the LIDAR range in the lineof-sight direction as a result of the stability of the time interval counter used to count the start/stop timing of the laser emission/reception. As the counter value increases with distance, the uncertainty of the counter accumulates as distance, and from the pre-flight test, this requirement (range uncertainty \pm 5.5 and $\pm 1 \mathrm{~m}$ at $20 \mathrm{~km}$ and $30-\mathrm{m}$ altitude, respectively) is satisfied. The in-flight data suggest that the actual accuracy is better than the pre-flight requirement. These numbers describe the total uncertainty of estimating spacecraft position with respect to the surface of the asteroid. 


\section{Evaluation of this method}

The advantage of the boulder identification method introduced in this study is that the field of view and boresight direction may be determined independently from the geodetic information of the target body or spacecraft position. For example, Matsumoto et al. (2020) used a shape model of the asteroid and spacecraft orbit, determined independently of LIDAR data. This means that for asteroid exploration missions, the shape and rotation axis may be obtained only after the spacecraft arrives at the target. Thus, the alignment direction of the altimeter with respect to the spacecraft cannot be determined using its geodetic information. However, the method we used is not always applicable as images and altimeter data must be collected simultaneously, and prominent surface features, such as boulders, must be present in an adequate configuration. Therefore, the laser link experiment, conducted for Hayabusa2 during the cruise phase, was still a good measure to determine the alignment when provided with an opportunity. The best method is the detection of the bright laser light footprints of an altimeter in an image directly using optical cameras that cover the same wavelength as the altimeter. It was possible to carry out such a method with the first Hayabusa mission (Abe et al. 2006), and it may be appropriate to consider future mission designs. As the method of this study may be applied with a relatively simple laser altimeter, such as Hayabusa2 LIDAR and a camera, it will be useful in future missions such as the Martian Moons eXploration (MMX) planned in 2024.

\section{Summary}

We determined the Hayabusa2 LIDAR boresight position in the ONC-T image using LIDAR time-series topographic data and prominent convex terrain features in camera images. Then, boresight direction was converted to alignment information with respect to the spacecraft body using the fitting position information of the ONC-T for the spacecraft. The boresight direction differed by approximately the same quantized uncertainty of $1 \mathrm{mrad}$ from the value determined using the laser link experiment in the winter of 2015. As the best-estimated field of view direction among a wider range of candidates was selected, the value in this study may be closer to reality. Additional laser link experiments with smaller scanning step sizes are likely to provide further constraints on the shape of the field of view. If we convert the uncertainty of the alignment direction of 4.6 pixels to the uncertainty of the spacecraft trajectory, this amounts to $0.4,2.1$, or $8.6 \mathrm{~m}$ in the cross- and/or along-track directions at altitudes of 1,5 , or $20 \mathrm{~km}$, respectively.

\section{Abbreviations}

APD: Avalanche photo diode; DEM: Digital elevation model; LIDAR: Light detection and ranging; ONC-T: Optical navigation camera-telescopic.

\section{Acknowledgements}

The authors are grateful to all Hayabusa2 team members, particularly JAXA personnel who were in charge of the daily spacecraft operations. The authors used the SPICE toolkit (Acton 1996) to convert LIDAR ranges to topography, and the Small Body Mapping Tool (Ernst et al. 2018) and SAO Image DS9 (http://ds9.si.edu/site/Home.html) for image processing. They would like to thank Editage (www.editage.com) for English language editing.

\section{Authors' contributions}

$\mathrm{HN}$ analyzed data and wrote the text. HN, HSe, KM, NN, TMi, YI, RY, KYa, FY, SA, $\mathrm{NarH}, \mathrm{NaOH}, \mathrm{AH}, \mathrm{HA}, \mathrm{SSa}, \mathrm{SO}, \mathrm{STs}, \mathrm{KA}$, and MS were responsible for science operation and data acquisition of LIDAR. SSu, RH, TMo, Ska, HSa, ET, CH, YY, MYa, TK, NS, HSu, KYoshio, MH, KO, YC, and MM were responsible for development, operation, data acquisition, and processing of ONC-T.YTs, SW, MYo, TS, STa, FT, S N, SKi, TY, NO, GO, YM, KYoshik, TT, YTa, and AF were responsible for critical spacecraft descent operations. All the authors read and approved the final manuscript.

\section{Funding}

This study was partly supported by Japan Society for the Promotion of Science (JSPS) Core-to-Core program "International Network of Planetary Sciences.

\section{Availability of data and materials}

Both LIDAR and ONC-T data will be open to public at the end of 2020 through JAXA's DARTS data archive system (http://darts.isas.jaxa.jp/planet/project/ hayabusa2/).

\section{Ethics approval and consent to participate}

Not applicable.

\section{Consent for publication}

All co-authors fully read the manuscript and agreed for submission.

\section{Competing interests}

The authors declare they have no competing interests.

\section{Author details}

${ }^{1}$ National Astronomical Observatory of Japan, Oshu, Iwate 023-0861, Japan. ${ }^{2}$ Chiba Institute of Technology, Narashino, Chiba 275-0016, Japan.

${ }^{3}$ National Astronomical Observatory of Japan, Mitaka, Tokyo 181-8588, Japan.

${ }^{4}$ Institute of Space and Astronautical Science, Japan Aerospace Exploration Agency, Sagamihara, Kanagawa 252-5210, Japan. ${ }^{5}$ University of Tokyo, Tokyo 113-0033, Japan. ${ }^{6}$ Nihon University, Funabashi, Chiba 274-8501, Japan. ${ }^{7}$ University of Occupational and Environmental Health, KitaKyushu, Fukuoka 807-8555, Japan. ${ }^{8}$ Kobe University, Kobe 657-8501, Japan. ${ }^{9}$ University of Aizu, Aizu-Wakamatsu 965-8580, Japan. ${ }^{10}$ Kochi University, Kochi 780-8520, Japan. 11 JAXA Space Exploration Center, Japan Aerospace Exploration Agency, Sagamihara, Kanagawa 252-5210, Japan. ${ }^{12}$ Rikkyo University, Tokyo 171-8501, Japan. ${ }^{13}$ National Institute of Advanced Industrial Science and Technology, Tokyo 135-0064, Japan. ${ }^{14}$ Osaka University, Toyonaka, Osaka 560-0043, Japan. ${ }^{15}$ Meiji University, Kawasaki, Kanagawa 214-8571, Japan. ${ }^{16}$ Instituto de Astrofisica de Canarias, University of La Laguna, E38205 Tenerife, Spain. ${ }^{17}$ Nagoya University, Nagoya, Aichi 464-8601, Japan. ${ }^{18}$ Mitsubishi Electric Corporation, Kamakura 247-8520, Japan. ${ }^{19}$ Research and Development Directorate, Japan Aerospace Exploration Agency, Sagamihara 252-5210, Japan. ${ }^{20}$ University of Tokyo, Kashiwa 277-8561, Japan. ${ }^{21}$ The Graduate University for Advanced Studies, SOKENDAl, Hayama 240-0193, Japan. 


\section{Appendix: Comparison with image data with Target Marker}

The Target Marker is a spherical object of approximately $10 \mathrm{~cm}$ in diameter and covered with a retroreflective sheet. It reflects light in the original direction when illuminated and is used as a bright visible mark when the spacecraft touches the surface of the asteroid. Two Target Markers (B and A) were dropped on October 25, 2018 and May 30, 2019, respectively, for two touchdown operations of the spacecraft. The Target Marker B was first dropped because of the location of the storage in the spacecraft. As the Target Marker may be clearly identified in ONC images, if it were detected on the ONC image and LIDAR at exactly the same time, these data would be very useful in determining alignment. Unfortunately, ONC-T images at these times were unavailable; therefore, data acquired close to the irradiation times were used for comparison.

When the transmitting laser of LIDAR irradiates a Target Marker, the returned laser intensity is one or more order of magnitude stronger than that of the surface. Therefore, at an altitude of $300 \mathrm{~m}$ or less, the inverse voltage of the detector (APD: avalanche photo diode) in the long-range receiving system is set to $0 \mathrm{~V}$ such that the electric avalanche is suppressed in the APD and the detector is undamaged. Instead, the short-range receiver is activated at this altitude for ranging. If the transmission light hits a Target Marker, the output power for the APD of the long-range receiver increases significantly because of the photovoltaic effect; this is similar to that of a solar cell. LIDAR illuminated these two Target Markers several times during the proximity phase, although only one case was obtained when both images and the LIDAR range were acquired almost simultaneously. The LIDAR irradiated Target Marker A for $2 \mathrm{~s}$ at $01 \mathrm{~h} 59 \mathrm{~m}$ $51.4 \mathrm{~s}$ and $01 \mathrm{~h} 59 \mathrm{~m} 52.4 \mathrm{~s}$ at an altitude of $75 \mathrm{~m}$ on June 13, 2019. The return pulse intensities as the APD output voltage were 805 and $477 \mathrm{mV}$, respectively, while the other values before and after were maintained at approximately $50 \mathrm{mV}$. The footprint size of the transmitting laser corresponding to a divergence angle of $2.4 \mathrm{mrad}$ was approximately $20 \mathrm{~cm}$ at this altitude. The moving speed of the Target Marker in the image was approximately one LIDAR footprint size per second; therefore, it was reasonable for it to be irradiated for $2 \mathrm{~s}$. On the other hand, the image data of the closest time were acquired at $01 \mathrm{~h} 59 \mathrm{~m} 49 \mathrm{~s}$ and $01 \mathrm{~h} 59 \mathrm{~m} 56 \mathrm{~s}$, meaning that no image was obtained when the LIDAR hit Target Marker A. Therefore, as a trial, the pixel coordinates of Target Marker A on the image when LIDAR hit was estimated by linear interpolation with these two images. The center coordinates of the Target Marker in these images are $(X, Y)=(638,494),(718,562)$. The estimated footprint

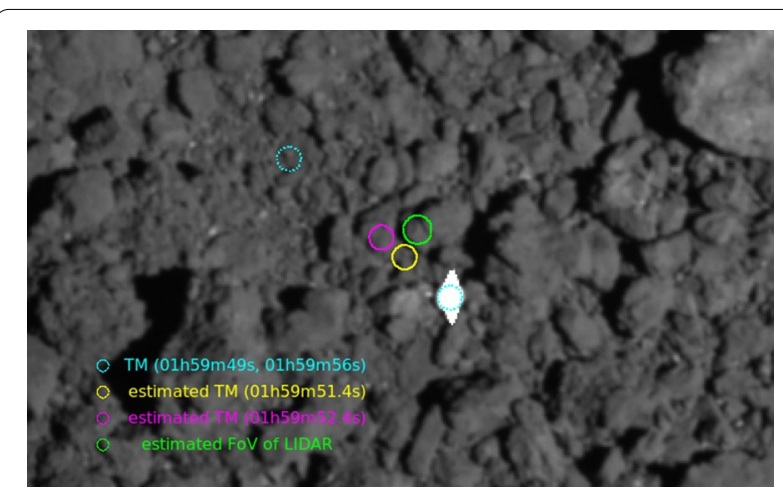

Fig. 12 Estimated Target Marker locations in the image at the shot time of 01 h 59 m 51 s and 01 h 59 m 52 s (yellow, magenta) by linear interpolation of Target Marker locations with two images and estimated LIDAR footprint position at 75-m altitude (green). Cyan dotted circles are observed location of the Target Marker in these two images. The white part in the center is the Target Marker in this image, but it looks elongated vertically due to saturation. Radius of these circles are 12 pixels, corresponding the viewing angle of the Target Marker A of $1.3 \mathrm{mrad}$ at altitude of $75 \mathrm{~m}$. (Image list: hyb2_ onc_20190613_015949_tvf_I2a.fit)

position of the LIDAR at the time of irradiation was obtained using altitude data where the alignment information obtained in the main text: this was $(X, Y)=(654.5$, 526.9). The results are shown in Fig.

12. The green circle indicates the estimated LIDAR footprint, while the yellow $((X, Y)=(660.9,513.4))$ and magenta $((X, Y)=(672.3,523.1))$ circles indicate the estimated Target Marker positions when the LIDAR irradiated it. The viewing angle of the $10 \mathrm{~cm}$ diameter Target Marker was $1.3 \mathrm{mrad}$ at an altitude of $75 \mathrm{~m}$, such that they were drawn as circles with a 12-pixel diameter. As a result, the estimated Target Marker position obtained by linear interpolation was displaced from the estimated LIDAR footprint by approximately one footprint from estimated Target Marker positions. The location of the first pulse was closer to the estimated field of view than the second pulse, as the APD output voltage implies.

As the estimated Target Marker position does not fall within the $1.5 \mathrm{mrad}$ field of view size, there may be a possibility that the LIDAR detected diffracted light of the return laser pulses outside the field of view of the LIDAR long-range telescope. As the return intensity is extremely strong because the laser was irradiated at a low altitude of $75 \mathrm{~m}$, it is not surprising that diffracted light with an intensity of approximately $1.7 \%$ (for the first bright ring) of the central part was detected. If further laser link experiments were carried out after the spacecraft returned to the Earth in the winter of 2020, 
it is expected that the width and shape of the field of view may be further investigated by scanning the spacecraft attitude with smaller step sizes than those used in the 2015 experiment.

Received: 10 March 2020 Accepted: 19 December 2020

Published online: 20 January 2021

\section{References}

Abe S, Mukai T, Hirata N, Barnouin-Jha OS, Cheng AF, Demura H, Gaskell RW, Hashimoto T, Hiraoka K, Honda T, Kubota T, Matsuoka M, Mizuno T, Nakamura R, Scheeres DJ, Yoshikawa M (2006) Mass and local topography measurements of Itokawa by Hayabusa. Science 312:1344-1347

Acton C (1996) Ancillary data services of NASA's navigation and ancillary information facility. Planet Space Sci 44:65-70

Araki H, Tazawa S, Noda H, Ishihara Y, Goossens S, Sasaki S, Kawano N, Kamiya I, Otake H, Oberst J, Shum CK (2009) Lunar global shape and polar topography derived from Kaguya-LALT laser altimetry. Science 323:897

Ernst CM, Barnouin OS, Daly RT, and the Small Body Mapping Tool Team (2018) The Small Body Mapping Tool (SBMT) for accessing, visualizing, and analyzing spacecraft data in three dimensions. LPSC abstract, no 1043

Goossens S, Matsumoto K, Rowlands DD, Lemoine FG, Noda H, Araki H (2011) Orbit determination of the SELENE satellites using multi-satellite data types and evaluation of SELENE gravity field models. J Geodesy 85:487-504

Matsumoto K, Noda H, Ishihara Y, Senshu H, Yamamoto K, Hirata N, Hirata N, Namiki N, Otsubo T, Watanabe S, Mizuno T, Yamamoto Y, Ikeda H, Ogawa N, Kikuchi S, Saiki T, Tsuda Y (2020) Improved trajectory of Hayabusa2 by combining LIDAR data and a shape model. Icarus 338:113574

Mizuno T, Kase T, Shiina T, Mita M, Namiki N, Senshu H, Yamada R, Noda H, Kunimori H, Hirata N, Terui F, Mimasu Y (2017) Development of the laser altimeter (LIDAR) for Hayabusa 2. Space Sci Rev 208:33-47

Noda H, Araki H, Goossens S, Ishihara Y, Matsumoto K, Tazawa S, Kawano N, Sasaki S (2008) Illumination conditions at the lunar polar regions by KAGUYA(SELENE) laser altimeter. Geophys Res Lett 35:24203

Noda H, Kunimori H, Mizuno T, Senshu H, Ogawa N, Takeuchi H, Moore C, Pollard A, Yamaguchi T, Namiki N, Kase T, Saiki T, Tsuda Y (2017) Laser link experiment with the Hayabusa2 laser altimeter for In-flight alignment measurement. Earth Planets Space 69:2 https://doi.org/10.1126/scien ce.aaz6306

Sugita S, Honda R, Morota T, Kameda S, Sawada H, Tatsumi E, Yamada M, Honda C, Yokota Y, Kouyama T, Sakatani N, Ogawa K, Suzuki H, Okada T,
Namiki N, Tanaka S, lijima Y, Yoshioka K, Hayakawa M, Cho Y, Matsuoka M, Hirata N, Hirata N, Miyamoto H, Domingue D, Hirabayashi M, Nakamura T, Hiroi T, Michikami T, Michel P, Ballouz RL, Barnouin OS, Ernst CM, Schröder SE, Kikuchi H, Hemmi R, Komatsu G, Fukuhara T, Taguchi M, Arai T, Senshu H, Demura H, Ogawa Y, Shimaki Y, Sekiguchi T, Müller TG, Hagermann A, Mizuno T, Noda H, Matsumoto K, Yamada R, Ishihara Y, Ikeda H, Araki H, Yamamoto K, Abe S, Yoshida F, Higuchi A, Sasaki S, Oshigami S, Tsuruta S, Asari K, Tazawa S, Shizugami M, Kimura J, Otsubo T, Yabuta H, Hasegawa S, Ishiguro M, Tachibana S, Palmer E, Gaskell R, Le Corre L, Jaumann R, Otto K, Schmitz N, Abell PA, Barucci MA, Zolensky ME, Vilas F, Thuillet F, Sugimoto C, Takaki N, Suzuki Y, Kamiyoshihara H, Okada M, Nagata K, Fujimoto M, Yoshikawa M, Yamamoto Y, Shirai K, Noguchi R, Ogawa N, Terui F, Kikuchi S, Yamaguchi T, Oki Y, Takao Y, Takeuchi H, Ono G, Mimasu Y, Yoshikawa K, Takahashi T, Takei Y, Fujii A, Hirose C, Nakazawa S, Hosoda S, Mori O, Shimada T, Soldini S, Iwata T, Abe M, Yano H, Tsukizaki R, Ozaki M, Nishiyama K, Saiki T, Watanabe S, Tsuda Y (2019) The geomorphology, color, and thermal properties of Ryugu: implications for parent-body processes. Science 364:eaaw0422

Suzuki H, Yamada M, Kouyama T, Tatsumi E, Kameda S, Honda R, Sawada H, Ogawa N, Morota T, Honda C, Sakatani N, Hayakawa M, Yokota Y, Yamamoto Y, Sugita S (2018) Initial inflight calibration for Hayabusa2 optical navigation camera (ONC) for science observations of asteroid Ryugu. Icarus 300:341-359

Watanabe S, Hirabayashi M, Hirata N, Na H, Noguchi R, Shimaki Y, Ikeda H, Tatsumi E, Yoshikawa M, Kikuchi S, Yabuta H, Nakamura T, Tachibana S, Ishihara Y, Morota T, Kitazato K, Sakatani N, Matsumoto K, Wada K, Senshu H, Honda C, Michikami T, Takeuchi H, Kouyama T, Honda R, Kameda S, Fuse T, Miyamoto H, Komatsu G, Sugita S, Okada T, Namiki N, Arakawa M, Ishiguro M, Abe M, Gaskell R, Palmer E, Barnouin OS, Michel P, French AS, McMahon JW, Scheeres DJ, Abell PA, Yamamoto Y, Tanaka S, Shirai K, Matsuoka M, Yamada M, Yokota Y, Suzuki H, Yoshioka K, Cho Y, Tanaka S, Nishikawa N, Sugiyama T, Kikuchi H, Hemmi R, Yamaguchi T, Ogawa N, Ono G, Mimasu Y, Yoshikawa K, Takahashi T, Takei Y, Fujii A, Hirose C, Iwata T, Hayakawa M, Hosoda S, Mori O, Sawada H, Shimada T, Soldini S, Yano H, Tsukizaki R, Ozaki M, lijima Y, Ogawa K, Fujimoto M, Ho TM, Moussi A, Jaumann R, Bibring JP, Krause C, Terui F, Saiki T, Nakazawa S, Tsuda Y (2019) Hayabusa2 arrives at the carbonaceous asteroid 162173 Ryugu--A spinning top-shaped rubble pile. Science 364:268-272

\section{Publisher's Note}

Springer Nature remains neutral with regard to jurisdictional claims in published maps and institutional affiliations.

\section{Submit your manuscript to a SpringerOpen ${ }^{\circ}$ journal and benefit from:}

- Convenient online submission

- Rigorous peer review

- Open access: articles freely available online

- High visibility within the field

Retaining the copyright to your article

Submit your next manuscript at springeropen.com 